\title{
Retroperitoneal Recurrence in Ovarian Sex Cord Stromal Tumor with Annular Tubules 16 Years Later.
}

\author{
Slimane Maher ${ }^{1}$,Hadidane Manel $^{1}$, Gadria Salma ${ }^{1}$,Bouzaiene Hatem ${ }^{1}$,Doghri \\ Raoudha $^{2}$,Ben Hassouna Jamel ${ }^{1}$, Mrad Karima $^{2}$,Rahal Khaled ${ }^{1}$. \\ ${ }^{1}$ Oncology Surgery Departement, ${ }^{2}$ immunohistopathology Departement, Salah Azaïz Institute, Tunis, Tunisia.
}

\begin{abstract}
:
Background: A sex cord tumor with annular tubules (SCTAT) is a rare but distinctive subtype of sex cord stromal tumor of the ovary. Its clinical features depend on an association with Peutz-Jeghers syndrome (PJS). Sctat not associated with PJS often manifests as a unilateral large mass and $20 \%$ of such tumors have malignant potential. Most patients with SCTAT are diagnosed at stage I, and metastasis is rare. Recurrence is not rare and ranges from 3 months to 20 years.

Case report: We represent a case of 26 years-old woman who had a history of a conservative surgery for Sctat of left ovary. 16 years later, she consulted for retroperitoneal mass that biopsy confirmed as a Sctat recurrence. Discussion and conclusion: Conservative surgery is the adequate treatment for SCTAT as well as it occur in young women to preserve fertility potential. Long-term follow-up is mandatory in such type of tumor because of the high risk of recurrence.
\end{abstract}

\section{Introduction}

Sex cord-stromal tumors (SCST) are rare ovarian tumors that represent approximately $7 \%$ of all ovarian malignancies [1]. These malignancies are often low grade and are frequently diagnosed in younger patients. Given these features and the rarity of these tumors, they are often diagnosed by pathology following surgery, which is often only a unilateral oopherectomy or ovarian cystectomy. While fertility sparing surgery is generally regarded as appropriate in apparently early stage disease, a staging procedure to include omentectomy, biopsies and pelvic and para-aortic lymph nodal dissection has been historically recommended for all patients with ovarian malignancies $[2,3]$.

Sex cord tumor with annular tubules (SCTAT) is a rare ovarian neoplasm that accounts for $6 \%$ of sex cord stromal tumors [4,5]. It was first described in 1970 by Scully, who characterized the tumor's unique histopathology consistent with coexistence of simple and complex annular tubules[6]. The predominant component of this tumor has intermediate morphologic features between granulosa cell tumors and Sertoli cell tumors and focal differentiation into either of these tumor types may occur [7].

The majority of SCTAT are benign neoplasms that arise sporadically in reproductive age patients. The most notable is a review of 74 cases in 1982, which reported that about one third of patients with SCTAT also have Peutz-Jeghers syndrome (PJS) [7]. Although most SCTATs associated with PJS are considered to be benign, rare instances of malignant behavior have been reported [8,9].

In women with non PJS associated SCTATs, malignant behavior with metastases may be seen in at least $20 \%$ of cases $[7,10,11]$.

Our aim is to describe the main features of recurrence of sex cord ovarian tumor with annular tubules.

\section{Case report :}

The patient was a woman aged 26 years old who consulted for 5-months history of amenorrhea without pelvic pain. Sixteen years before, she had a left salpingo-oophorectomy and ipsilateral pelvic node sampling for surgical stade IA FIGO ovarian sex cord tumor with annular tables. No adjuvant treatment was administrated. The patient was lost of view and consulted us 16 years later for amenorrhea. An abdominopelvic mobile left mass of $20 \mathrm{~cm}$ was palpated without any associated lymph nodes. Thoraco-abdomino-pelvic computed tomography revealed a solid heterogenous well limted mass with calcifications situated in the left retroperitoneal region, measuring $16 \times 14 \times 10 \mathrm{~cm}$. The mass repulsed the left kidney and compressing left ureter with left hydronephrosis. No signs of invasion of the adjacent organs were noted (figure 1) . A scanno-guided biopsy of the mass was realised.

The histology examination (figure 2a, 2b) revealed tumor proliferation with annular, alveolo-trabecular structures. Tumor cells had eosinophile granular cytoplasm with round or ovoid nucleus containing pale chromatin. The cells were arranged into peripheral placement. Hyaline and calcifications deposits were present as well as disseminated necrosis sites. The diagnosis of SCTAT recurrence was evoqued. 
Laboratory testing showed a high anti mullerian hormone (AMH) level at $5817 \mathrm{ng} / \mathrm{ml}$. Serum inhibinB level was $>5000 \mathrm{pg} / \mathrm{ml}$. Serum levels of CA125 tumor antigen, $\alpha$-feotoprotein and sex hormone-biding globulin were normal. Estradiol was not measured. The patient was operated. We discovered in laparotomy a voluminous retropertoneal left encapsulated mass, with smooth limits, measuring $20 \times 15 \mathrm{~cm}$.

The tumor was placed over aorta and intimately attached to the left renal vein, tail of pancreas without invasion of these structures. The left mesocolon was massively accolated to the mass. The right ovary, uterus and liver were normal. No ascites was objectified. We realized en bloc resection of the tumor and left segmental colon (figure 3).We did para-aortic lymphadenectomy because the presence of palpable nodes in this region. After definitive histological exam of the whole mass, no glanglial parenchyma was noted.

Her post operative course was remarquable by menses returns, 3 days after surgery. AMH and Inhibine-B level had decreased post operatively at $2,44 \mathrm{ng} / \mathrm{ml}$ and $<8 \mathrm{ng} / \mathrm{ml}$ respectively. Multidisciplinary medical committee decided to start an adjuvant chemotherapy based on bleomycin, etoposide and cis-platinium. Before that, ovarian stimulation was managed but didn't show hormonal activity. Fertility preservation attempt was a failure. The patient is receiving chemotherapy.

\section{Discussion}

Sex cord-stromal tumors are rare ovarian neoplasms that are often unsuspected and incompletely staged at the time of primary surgery. These malignancies are typically diagnosed at an apparently early stage and generally have a favorable prognosis. Sex cord tumor with annular tubules (SCTAT) is a rare ovarian neoplasm that accounts for $6 \%$ of sex cord stromal tumors [4,5]. The predominant component of this tumor has intermediate morphologic features between granulosa cell tumors and Sertoli cell tumors and focal differentiation into either of these tumor types may occur [7].

There are two forms of SCTAT: with PJS, in which the tumors usually consist of small, bilateral, multifocal, calcified lesions scattered within the ovarian stroma, and without PJS, in which the tumors generally present as large, unilateral, solitary, non calcified neoplasms [7]. Concerning patients without PJS (sporadic SCTAT) $21.9 \%$ cases in literature were clinically malignant [7]. Our patient showed no manifestations of PJS. The most frequent clinical manifestations of SCTAT relate to estrogen production. Consequently, children with SCTAT may present with signs of precocious puberty. This was not the situation of our patient

The diagnosis of SCTAT is based on histologic findings of simple and complex annular tubules encircling central hyaline masses, as it was objectified with our patient.

The malignant potential of SCTAT cannot be reliably predicted by microscopic examination of the primary tumor $[12,13,14]$. The serum tumor marker inhibin as well as some of immunohistochemical markers (inhibin, MIS, CD99, estrogen receptor, progesterone receptor, and androgen receptor) have been studied as diagnostic tools and predictors of clinical behavior of the tumor postoperatively [10]. Non-PJS-associated SCTATs also commonly secrete estrogen and progesterone, although the sensitivity of following serum markers of these hormones is low, especially when residual tumor volume is limited [15]. Inhibin a heterodimeric protein hormone secreted by granulosa cells that acts as an inhibitor of follicle-stimulating hormone(FSH) synthesis, has been known to be a sensitive immunohistochemical marker in diagnosing sex cord stromal tumors.

Clinical management is surgical and should include ovarian cystectomy versus oophorectomy, depending on the patient's age, the degree of ovarian involvement, and the desire to preserve ovarian function of the affected ovary. Fertility sparing surgery is often desired among these women.Thus gynecologic oncologists are frequently faced with decisions regarding the need for additional staging procedures in this patient cohort of reproductive age women. These tumors were not noted to spread to pelvic or para-aortic lymph nodes as there were no involved nodes observed among any of the 47 women that had nodal tissue evaluated in Brown et al study [16].Thrall et al support the rarity of lymph node involvement in SCST and support the hypothesis that the routine removal of pelvic and para-aortic lymph nodes may be omitted from the surgical staging of this malignancy [17]. Moreover, a recent retrospective study by Yinon et al demonstrated no statistically significant difference in tumor recurrence rates between patients who had undergone cystectomy versus a unilateral salpingo-oophorectomy [18].

The effects of chemotherapy and radiotherapy are not clear. Further studies are needed to confirm the effects of chemotherapy and radiotherapy. The time of first recurrence in cases of malignant SCTAT has ranged from 3 months to 20 years, and six patients reportedly have died of recurrence $[13,14,16]$. Malignant SCTAT seems to spread mainly via the lymphatics with typical sites of tumor metastasis being the pelvic, para-aortic, and supraclavicular lymph nodes. Other sites of tumor recurrence and metastasis include the retroperitoneum, parietal and visceral peritoneum liver, kidney, and lung [13,14,16].

In Thrall et al study, among patients with recurrences, 2 were noted to have isolated retroperitoneal disease recurrence at 28 and 66 months [17]. Neither of these women had a lymph node dissection at the time of primary recurrence. Furthermore, tumor size was found to be the most important predictor of death from 
disease. Nodal involvement at the time of recurrent disease was documented in $5 \%$ of cases in another series [18].

No disease recurrences were noted among women with primary tumors less than $7 \mathrm{~cm}$. Tumor size has been noted to be an important prognostic factor in many reports [19,20,21], and careful evaluation of women with larger lesions, especially over $7 \mathrm{~cm}$ is warranted. In these series, tumors greater than $10-15 \mathrm{~cm}$ were associated with an increased risk of recurrence and death from disease. Stage has been previously demonstrated to be one of the most important predictors of survival [22,23]. Age has not been consistently correlated with survival among women with SCST [19,21,24,25]. Vascular space invasion by individual tumor cells and tumor cell clusters has been reported in two cases of recurrent SCTAT, one of which also had infiltrated the capsule [26].

Recurrent patients would present with menstrual disturbances and pelvic or abdominal masses again at the time of recurrence. Thus, careful history taking and physical examination combined with imaging tests can be used as monitoring methods. In addition, both serum estradiol and progesterone can be used as tumor markers during follow-up. Elevated serum inhibin level was also reported in patients with SCTAT by several researchers $[27,28]$. Serum inhibin may also be used for follow-up. Preoperative evaluation is very important for recurrent SCTAT. PET-CT scan is a favorable method for the evaluation of tumor size, numbers, location of recurrent tumors, and the therapeutic effect. Fertility-sparing surgery can be still considered for patients with no involvement of controlateral ovary and uterus. As in our patient, fertility-sparing surgery complete recurrence resection was possible. Because the majority of recurrent tumors located in retroperitoneum, particularly in pelvic lymph nodes ipsilateral to the primary tumor and/or para-aortic lymph nodes, ipsilateral pelvic and paraaortic lymphadenectomy may be considered to reduce recurrence.

The prognosis is excellent because most of patients have stage I disease at presentation as our patient.

\section{Conclusion}

SCTAT diagnosis is usually based on pathological examination of the tumor and typical clinical presentations are usually consistent with a hormone-secreting tumor, resulting in precocious puberty in children or menstrual disturbances, such as amenorrhea, in reproductive age women. Histologically, SCTAT contains features similar to GCT (Granulosa celle tumor) and Sertoli cell tumors. There is no standard treatment protocol for patients with SCTAT because of its rarity.

Initial management for patients with SCTAT is surgery based on preserving ovarian tissue for fertility because most cases occur in adolescents or in reproductive age women, but this should be weighed against an almost $20 \%$ risk of malignant transformation. Although SCTAT prognosis is relatively favorable, the risk of recurrence is still very high. Long-term follow-up is essential for patients with SCTAT.

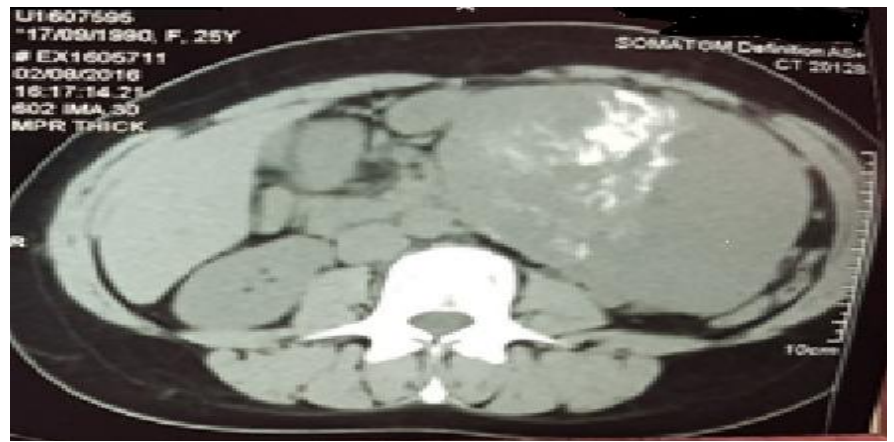

Figure 1: CT imaging, frontal slice of the retroperitoneal recurrence: heterogenous solid mass with calcifications

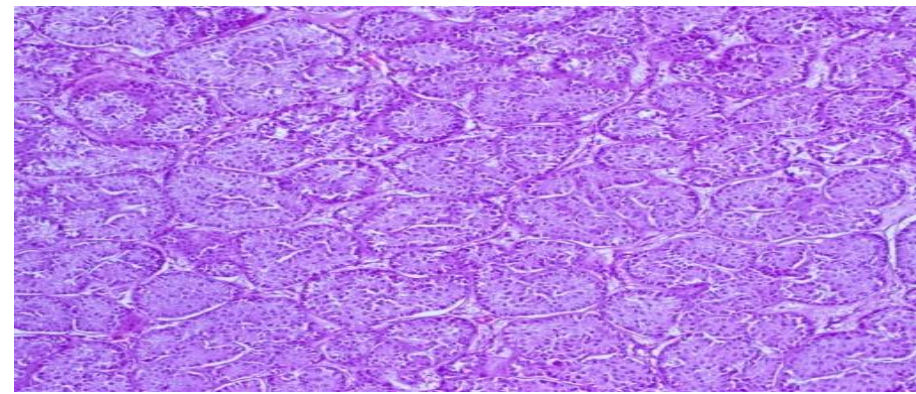

Figure 2, a: Histologic features of Sex cord stromal tumor with annular tubules of the ovary recurrence (Hematoxylin and Eosin x10) 


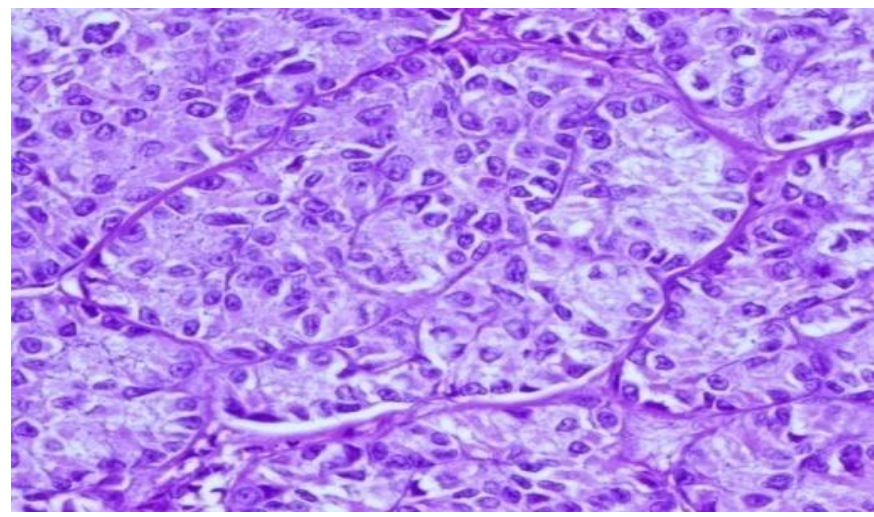

Figure 2, b: Histologic features of Sex cord stromal tumor with annular tubules of the ovary recurrence (H\&E $\mathrm{x} 20$ )

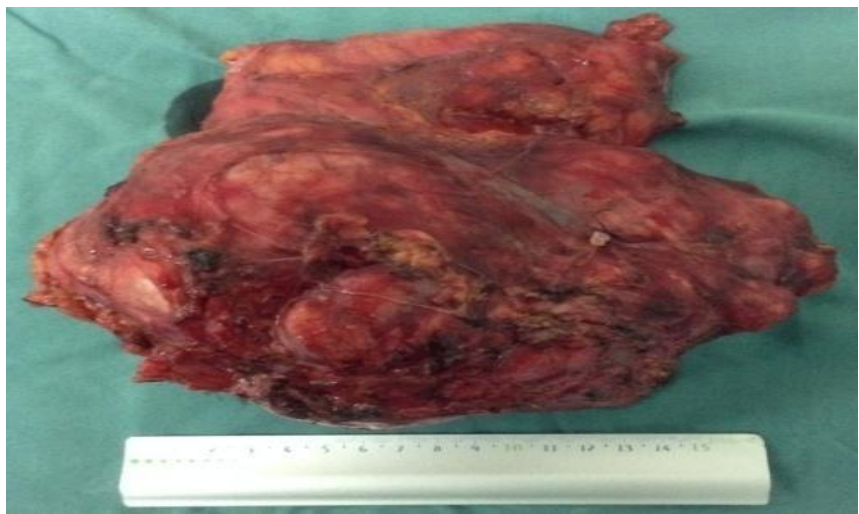

Figure 3 : Macroscopic view of the retroperitoneal recurrence.

\section{References}

[1]. Koonings PP, Campbell K, Mishell DR Jr. Grimes DA. Relative frequency of primary ovarian neoplasms: a 10-year review. Obstet Gynecol. 1989; 74:921-6.

[2]. NCCN. Practice guidelines in Oncology v.2.2011 Ovarian Fallopian Tube and Primary Peritoneal Carcinomas. 2011.

[3]. Gershenson DM. Management of early ovarian cancer: germ cell and sex cord-stromal tumors. Gynecol Oncol. 1994; 55:S62-72.

[4]. Scully RE, Sobin LH. Histological typing of ovarian tumors. In: (World Health Organization international histological classification of tumors.). 2nd ed. Berlin: Springer, 1999.

[5]. ZumkellerW, Krause U, Holzhausen HJ, HirschW, Finke R, Burdach S. Brief report—ovarian sex cord tumor with annular tubules associated with precocious puberty. Med Pediatr Oncol 2000;35:144-6.

[6]. Scully RE. Sex cord tumor with annular tubules: a distinctive ovarian tumor of the Peutz-Jeghers syndrome. Cancer 1970;25:110721.

[7]. Young RH, Welch WR, Dickersin GR, Scully RE. Ovarian sex cord tumor with annular tubules: review of 74 cases including 27 with Peutz- Jeghers syndrome and 4 with adenoma malignum of the cervix. Cancer 1982;50:1384-402.

[8]. Ayadi-Kaddour A, Bouraoui S, Bellil K, Bellil S, Kchir N, Zitouna MM et al. Colonic adenocarcinoma and bilateral malignant ovarian sex cord tumor with annular tubules in Peutz-Jeghers syndrome. Pathologica 2004;96:117-20.

[9]. Lele SM, Sawh RN, Zaharopoulos P, Adesokan A, Smith M, Linhart JM, et al. Malignant ovarian sex cord tumor with annular tubules in a patient with Peutz-Jeghers syndrome: a case report. Mod Pathol 2000;13: 466-70.

[10]. Roth LM. Recent advances in the pathology and classification of ovarian sex cord stromal tumors. Int J Gynecol Pathol 2006;25:199-215.

[11]. Crain JL. Ovarian sex cord tumor with annular tubules: steroid profile. Obstet Gynecol 1986;68(3 Suppl):75S-9S.

[12]. Gloor E. Ovarian sex cord tumor with annular tubules. Virchows Arch A 1979;384:185-93.

[13]. Ahn GH, Chi JG, Lee SK. Ovarian sex cord tumor with annular tubules. Cancer 1986;57:1066-73.

[14]. Gustafson ML, Lee MM, Scully RE, Moncure AC, Hirakawa T, Goodman A, et al. Mullerian inhibiting substance as a marker for ovarian sex cord tumor. N Engl J Med 1992;326:466-71.

[15]. Scully RE. Hormonally active ovarian tumors. Verh Deutsch Ges Pathol 1997;81:245-52.

[16]. Brown J, Sood AK, Deavers MT, Milojevic L, Gershenson DM. Patterns of metastasis in sex cordstromal tumors of the ovary: Can routine staging lymphadenectomy be omitted? Gynecol Oncol. 2009; 113:86-90.

[17]. Thrall MM, Paley P, Pizer E, Garcia R, Goff BA. Patterns of spread and recurrence of sex cord-stromal tumors of the ovary. Gynecol Oncol. 2011 August ; 122(2): 242-245.

[18]. Yinon Y, Beiner ME, Gotlieb WH, Korach Y, Perri T, Ben-Baruch G. Clinical outcome of cystectomy compared with unilateral salpingo-oophorectomy as fertility-sparing treatment of borderline ovarian tumors. Reprod Surg 2007;88:479-84.

[19]. Chan JK, Zhang M, Kaleb V, Loizzi V, Benjamin J, Vasilev S, Osann K, Disaia PJ. Prognostic nfactors responsible for survival in sex cord stromal tumors of the ovary—a multivariate analysis. Gynecol Oncol. 2005; 96:204-9.

[20]. Fox H, Agrawal K, Langley FA. A clinicopathologic study of 92 cases of granulosa cell tumor of the ovary with special reference to the factors influencing prognosis. Cancer. 1975; 35:231-41. 
[21]. Evans AT 3rd, Gaffey TA, Malkasian GD Jr. Annegers JF. Clinicopathologic review of 118 granulosa and 82 theca cell tumors. Obstet Gynecol. 1980; 55:231-8.

[22]. Zhang M, Cheung MK, Shin JY, Kapp DS, Husain A, Teng NN, Berek JS, Osann K, Chan JK. Prognostic factors responsible for survival in sex cord stromal tumors of the ovary--an analysis of 376 women. Gynecol Oncol. 2007; 104:396-400. [PubMed: 17030354]

[23]. Zanagnolo V, Pasinetti B, Sartori E. Clinical review of 63 cases of sex cord stromal tumors. Eur J Gynaecol Oncol. 2004; 25:431-8. [PubMed: 15285297]

[24]. Tavassoli FA, Norris HJ. Sertoli tumors of the ovary. Cancer 1980;46:2281-97.

[25]. Lele SM, Sawh RN, Zaharopoulos P, Adesokan A, Smith M, Linhart JM, et al. Malignant ovarian sex cord tumor with annular tubules in a patient with Peutz-Jeghers syndrome: a case report. Mod Pathol. 2000;13(4):466-70.

[26]. Bjorkholm E, Silfversward C. Prognostic factors in granulosa-cell tumors. Gynecol Oncol. 1981;11:261-74.

[27]. Nosov V, Park S, Rao J, Memarzadeh S. Non-Peutz-Jeghers syndrome associated ovarian sex cord tumor with annular tubules: a case report. Fertil Steril. 2009;92(4):e1495-97.

[28]. Bercaw JL, Sanchez J, Byrd RH, Bhattacharjee MB, Dietrich JE. Sex cord tumor with annular tubules in a young adolescent with Von Willebrand's disease. J Pediatr Adolesc Gynecol. 2010;23(3):e111-4. 